EPJ Web of Conferences 93,01003 (2015)

DOI: $10.1051 /$ epjconf/20159301003

(C) Owned by the authors, published by EDP Sciences, 2015

\title{
Complementarity in nuclear structure studies
}

\author{
P.E. Garrett ${ }^{1, a}$ \\ ${ }^{1}$ Department of Physics, University of Guelph, Guelph, ON, N1G2W1, Canada
}

\begin{abstract}
The nuclear many-body system displays a wide variety of behaviors, including collective and singleparticle degrees of freedom. Specific reactions may probe these different aspects, the data from which may both complement and also appear to be in conflict with each other. In the present work, examples are given where information from one experiment enhances and reinforces the information from another, and also an example where the data appear to be in conflict and result in a re-interpretation of structure.
\end{abstract}

\section{Introduction}

The atomic nucleus enjoys a rather unique place in physics. A many-body fermionic system with two basic building blocks, it exhibits incredibly complex excitations. Compared with molecular systems, for example, where rotational, vibrational, and electronic excitation energy scales differ by one to two orders of magnitude, in the nuclear system the energy scales are approximately equal. Because of this, very detailed spectroscopy is required to determine the nature of the excitation; the excitation energy alone is insufficient. Fortunately, we have at our disposal a wide variety of probes that can be used, some of which are highly selective and directly probe the components in the wave function, such single- and twonucleon-transfer reactions, high-energy inelastic hadronic scattering, etc., and others that are insensitive to the details of the nuclear wave functions, such as fusion-evaporation reactions and low-energy inelastic scattering.

Nuclei lying on or near the valley of stability can typically be studied by the variety of techniques mentioned above so that a large complementary set of data can be obtained. The complementary data can shed light on both collective, pairing, and single-particle aspects of the excitations, although sometimes in a contradictory way. While the contradictions can appear at first to be puzzling, the full scope of information ultimately leads to a deeper understanding of excited states in nuclei. Further, the correct interpretation of excited states in nuclei near stability is vital to serve as benchmarks when attempting to elucidate structure in nuclei far from stability. In the following, I give some examples of the usefulness of complementary data in working out electromagnetic transition matrix elements, namely combining data from experiments that measure level lifetimes or matrix elements via Coulomb excitation, and experiments that achieve high-sensitivity to weak decay branches. Further, an example is given of

\footnotetext{
a e-mail: pgarrett@physics.uoguelph.ca
}

the contradictory conclusions that may be reached regarding the nature of excited states when viewed with different probes.

\section{Complementarity in electromagnetic matrix elements}

Electromagnetic matrix elements provide critically important information for nuclear structure. Direct lifetime measurements can be obtained via electronic timing techniques, or Doppler-shift techniques. Once the lifetime is known, the matrix elements can be calculated through knowledge of the $\gamma$-ray transition branches. Coulomb excitation, on the other hand, provides direct information on the matrix elements through the measurements of the $\gamma$-ray yields. Provided that the reaction takes place in a "safe" manner, calculations of the well-known electromagnetic interaction can be performed that are effectively exact, even if carried out in a semi-classical approximation.

One of the issues that Coulomb excitation may suffer from arises from ambiguities in the population pathways. For example, a high-lying $4^{+}$state may be reached via a direct $E 4$ transition from the ground state, via successive $E 2$ transitions in a two-step excitation involving the first $2^{+}$state, the second $2^{+}$state, etc., or any other multi-step combination provided that the angular-momentum/parity selection rules are obeyed. Typically, when going beyond all but the lowest excitations these ambiguities greatly increase the uncertainties on the extracted matrix elements. However, these can be overcome provided that: a) there are sufficiently large number of complementary data in the form of already known level lifetimes, transition branching ratios, etc., or b) the Coulomb-excitation experiment is performed under a variety of conditions, such as changing projectiles, measuring yields as a function of the scattering angle, etc.

Much of the Coulomb excitation data for stable nuclei currently available was obtained in the late 1960's 

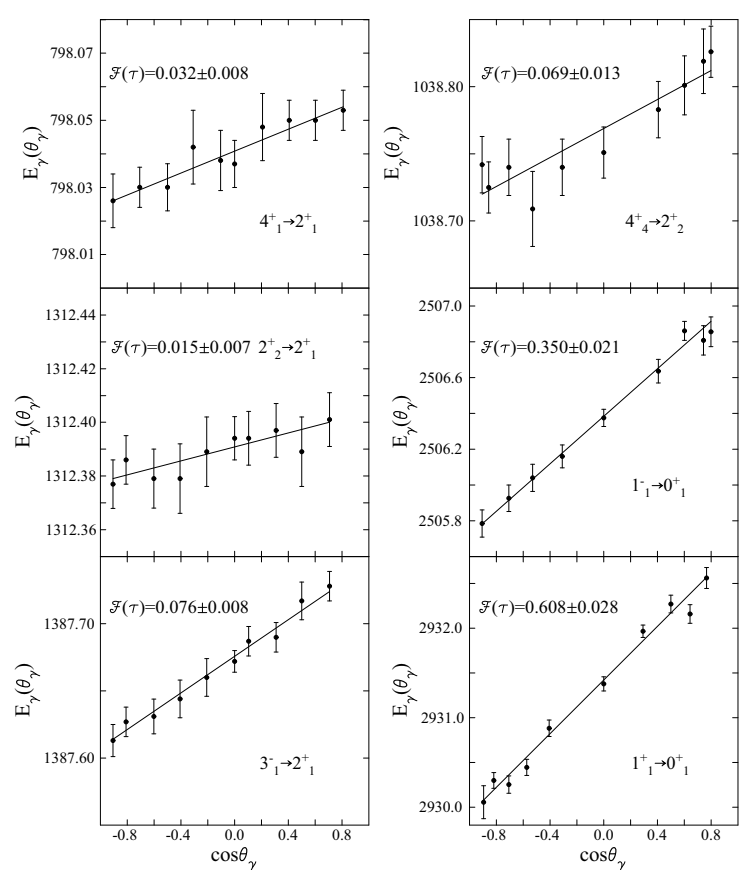

Figure 1. Examples of Doppler shifts from the ${ }^{112} \mathrm{Cd}\left(n, n^{\prime} \gamma\right)$ reaction. From data like these, using knowledge of the stopping powers the level lifetimes can be extracted. A major advantage of the $\left(n, n^{\prime} \gamma\right)$ reaction with accelerator-produced nearly monoenergetic neutrons is the ability to choose the maximum excitation energy of the nucleus, and hence control the feeding to a state of interest.

and early 1970 's, often using small volume Ge(Li) detectors or NaI detectors. Using the stable even-even $\mathrm{Cd}$ isotopes as an example, despite their pivotal role serving as a benchmark for multi-phonon vibrational excitations (see e.g. Kern et al. [1]), only in ${ }^{114} \mathrm{Cd}[2]$ are there an extensive set of matrix elements from data obtained using a modest setup of NaI, Ge(Li), and PPAC detectors. The remaining stable even-even $\mathrm{Cd}$ isotopes have been examined in Coulomb excitation using NaI detectors (see, e.g. Ref. [3]). The Coulomb excitation data [3] for the series of $\mathrm{Cd}$ isotopes was crucial in establishing the validity of the multi-phonon vibrational picture, but the more extensive data on ${ }^{114} \mathrm{Cd}$, which raised serious questions regarding its structure, remained isolated in the sense that it was never followed up; despite the extraction of large values of $\langle\beta\rangle$ for the ${ }^{114} \mathrm{Cd} 0^{+}$states through the use of the Kumar-Cline sum rules, probing Coulomb excitation experiments on the other Cd isotopes were not performed. The lack of highprecision and extensive data, together with the apparent success of models like the IBM incorporating mixing with the deformed intruder bands $[4,5]$ that could reproduce the set of $B(E 2)$ values and level energies as known at the time, created the persistent impression that the mid-shell even-even $\mathrm{Cd}$ isotopes were, in fact, paradigms of vibrational motion [1].

Beginning in the mid-1990's, an extensive program of investigation of the stable even-even $\mathrm{Cd}$ isotopes was initiated at the University of Kentucky accelerator laboratory using the powerful $\left(n, n^{\prime} \gamma\right)$ reaction. This reaction, which for low neutron energies of several $\mathrm{MeV}$ is dominated by the compound nucleus reaction, has the advantage of high cross section for low-spin states, including spin 0 , and observable Doppler shifts for states with lifetimes up to approximately 1 ps. Taking advantage of either the ${ }^{3} H(p, n)$ or $d(d, n)$ reactions to produce nearly mono-energetic neutrons, the maximum excitation energy of the target nucleus can be chosen in such as way as to minimize the problematic feeding issue that plagues Doppler-shift analysis in charged-particle fusion-evaporation reactions. Figure 1 shows some examples of Doppler shifts extracted from the ${ }^{112} \mathrm{Cd}\left(n, n^{\prime} \gamma\right)$ reaction. The Doppler shift of the $798-\mathrm{keV}$ $\gamma$ ray, which is the $4_{1}^{+} \rightarrow 2_{1}^{+}$transition in ${ }^{112} \mathrm{Cd}$, is a mere $25 \mathrm{eV}$ from the most forward to the most backward angles measured; very high statistics combined with a large dispersion for the ADC (i.e. small keV per channel) and the use of radioactive sources placed in view of the hpGe detectors to provide for continuous monitoring for gain shifts as a function of angle enable the extraction of such small energy shifts. Knowledge of the stopping power then allows for the determination of the level lifetime [6]. The 798-keV transition shown in Fig. 1 has a Doppler shift corresponding to $\tau=1000_{-340}^{+1000} \mathrm{fs}$, in excellent agreement with the literature value of $\tau=1300 \pm 100 \mathrm{fs}$ [7].

The first nucleus of the series of even-even $\mathrm{Cd}$ isotopes to be studied with the $\left(n, n^{\prime} \gamma\right)$ reaction, ${ }^{112} \mathrm{Cd}$, was initially interpreted [8] in terms of the vibrational limit of the IBM. Soon thereafter, measurements $[9,10]$ on ${ }^{110} \mathrm{Cd}$ were also viewed as being in good agreement with the vibrational model. However, a study [12] of ${ }^{116} \mathrm{Cd}$ found that the decays of the $0_{2}^{+}$and $0_{3}^{+}$states could not be explained in the usual strong-mixing scenario $[4,11]$; the decay of the intruder band head (the $0_{3}^{+}$state in ${ }^{116} \mathrm{Cd}$ ) remained enhanced over the decay of the $0^{+}$"two-phonon" state, despite the latter being lower in energy. A re-examination of the $\left(n, n^{\prime} \gamma\right)$ results for ${ }^{112} \mathrm{Cd}$ with a more extensive analysis found serious discrepancies between the experimental data and IBM-2 calculations for the three-phonon levels [13], as did a study of ${ }^{114} \mathrm{Cd}$ [14]. In fact, while in any one particular nucleus the discrepancies might not be viewed as serious, it was realized that the discrepancies occurred systematically across the mid-shell Cd isotopes [15], and thus unlikely to be due to mixing.

While the $\left(n, n^{\prime} \gamma\right)$ reaction provides a wealth of spectroscopic information, including the level lifetimes or limits for most of the levels observed [10,13,14], due to the backgrounds present in the in-beam studies and the large sample sizes required for the inelastic neutron scattering, it is not particularly sensitive to low-energy transitions between highly-excited states. These transitions are often the most important ones to properly characterize the collectivity, but also very often have branching ratios below the $10^{-3}$ level. Therefore, further experiments that are sensitive to particularly weak, low-energy transitions need to be performed. It was for this reason that a program pursuing high-statistics $\beta$-decay measurements was initiated.

The program at the TRIUMF-ISAC facility to study $\beta$ decays feeding the $\mathrm{Cd}$ isotopes used the $8 \pi$ spectrometer 


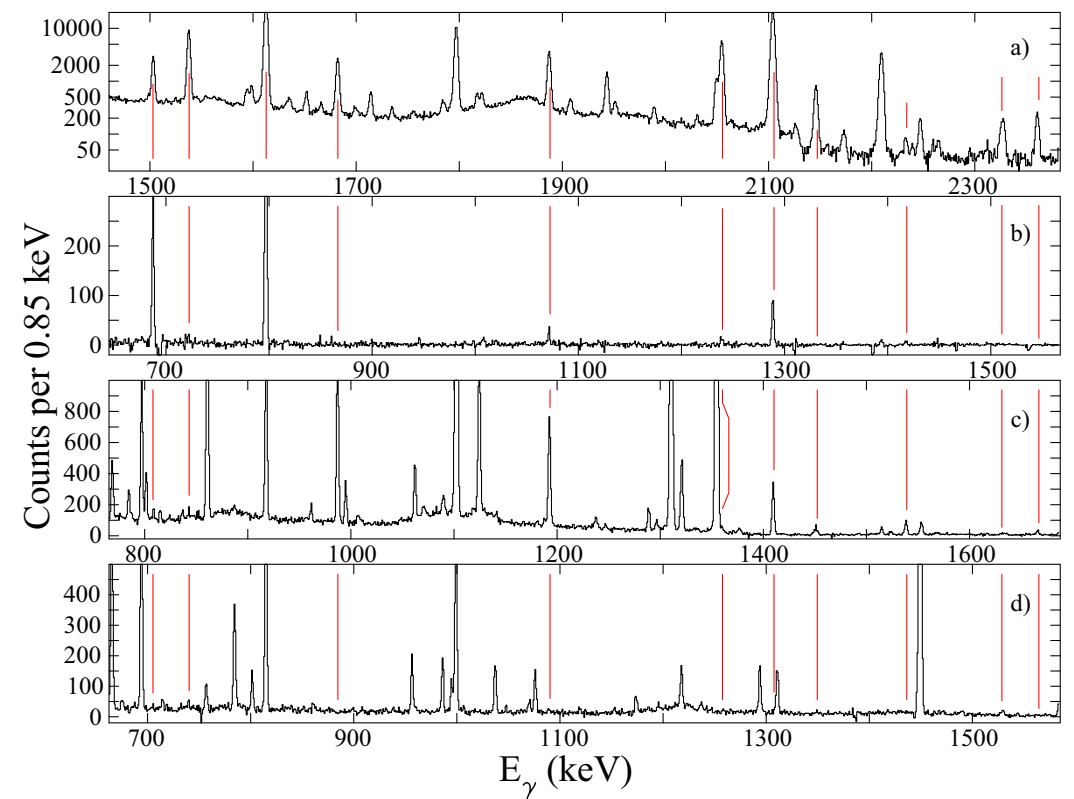

Figure 2. Examples of coincidence spectra obtained from the decay of ${ }^{112} \mathrm{In} /{ }^{12} \mathrm{Ag} \rightarrow{ }^{112} \mathrm{Cd}$ with the $8 \pi$ spectrometer. The spectrum in panel a) results from a coincidence gate on the $617-\mathrm{keV} 2_{1}^{+} \rightarrow 0_{1}^{+}$transition. Transitions from all known $2^{+}$levels between $2 \mathrm{MeV}$ and $3 \mathrm{MeV}$ are marked with a vertical line. The spectrum in panel b) is obtained with a coincidence gate on the $816-\mathrm{keV} 0_{3}^{+} \rightarrow 2_{1}^{+} \gamma$ ray, where the vertical lines guide the eye (where necessary) to locations for an expected transition from the $2^{+}$states identified with their energies. The spectrum in panel c) is obtained with a coincidence gate on the $695-\mathrm{keV} 2_{2}^{+} \rightarrow 2_{1}^{+} \gamma$ ray, and that in panel d) is obtained with a coincidence gate on the $798-\mathrm{keV} 4_{1}^{+} \rightarrow 2_{1}^{+} \gamma$ ray. and its auxiliary devices. The $8 \pi$ spectrometer consisted of $20 \mathrm{hpGe}$ detectors equipped with BGO anti-Compton shields surrounding a target chamber with a radius of 11 $\mathrm{cm}$. Internal to the vacuum is a tape on which the beam is deposited at the focus of the array, and which can be moved in a controlled manner to transport the activity out of the target chamber. Also present inside the target chamber are optional auxiliary detectors consisting of 20 thin plastic scintillators for $\beta$-particle tagging, or $5 \mathrm{Si}(\mathrm{Li})$ detectors for conversion electron studies. The power of the $8 \pi$ spectrometer is aptly demonstrated in Fig. 2 that shows a series of $\gamma$-ray coincidence spectra with gates on selected $\gamma$-rays following the $\beta$ decay of a beam of ${ }^{112} \mathrm{In}$ and ${ }^{112} \mathrm{Ag}$.

When seeking evidence for the existence of multiphonon structures, as the energy and phonon number increases, the increasing level density would be expected to fragment the collective wave function, potentially over many states. Thus, failure to locate the expected E2 strength in one particular state may not necessarily indicate a lack of existence of the multi-phonon excitation. However, with the highly sensitive data from the $\beta$ decay, and the knowledge that all known states of a particular spin up to a certain energy are populated, the sum of the observed (or upper limit) of the E2 strength can be constructed. From the data like those presented in Fig. 2, combined with the lifetimes from the $\left(n, n^{\prime} \gamma\right)$ experiment, the $B(E 2)$ for all decays into specific final states can be examined. This is shown in Fig. 3 for ${ }^{112} \mathrm{Cd}$ for decays from high-lying $2^{+}$states into the hypothesized twophonon states; while there would be agreement for decay into the purported $0^{+}$two-phonon state, the strength for decay into the $2^{+}$two-phonon is much weaker than expected, and there is no decay, and a severe upper limit on its possibility, into the $4^{+}$two-phonon state. It can thus be concluded that the $2^{+}$three phonon strength is not fragmented - it does not exist.
Recently, a series [16-20] of Coulomb excitation experiments were performed with GAMMASPHERE in inverse kinematics of $\mathrm{Xe}$ beams on $\mathrm{C}$ targets. The aims of these experiments were to determine the $B(E 2)$ values for excitation to low-lying $2^{+}$states, and an extensive set of matrix elements for each nucleus was determined. These measurements convincingly demonstrated the power of modern spectrometers to extract multiple, high-precision matrix elements through Coulomb excitation, and the majority of them were completed with less than a day, and in some cases only 12 hours, of beam time.

Concentrating on ${ }^{124} \mathrm{Xe}$, the Coulomb excitation experiment [16] extracted matrix elements for excitation of the $2^{+}$states in the $K^{\pi}=0_{2}^{+}$and $0_{3}^{+}$bands. The $2^{+} \rightarrow$ $0^{+}$transitions in these bands, however, were not accurately determined; for the $K^{\pi}=0_{2}^{+}$band the extracted $B\left(E 2 ; 2^{+} \rightarrow 0^{)}\right.$value was $62 \pm 36$ W.u. As in the case of the $\mathrm{Cd}$ isotopes, $\beta$-decay is an ideal tool to observe the weak, low-energy branches. Using Cs beams from the TRIUMF-ISAC facility, the $\beta$-decay of ${ }^{124} \mathrm{Cs}$ was studied with the $8 \pi$ spectrometer. Shown in Fig. 4 is a portion of the $\gamma$-ray coincidence spectrum for a gate taken on the $914-\mathrm{keV} 0_{2}^{+} \rightarrow 2_{1}^{+} \gamma$ ray. The observed $360-\mathrm{keV} \gamma$-ray is the $2^{+} \rightarrow 0^{+}$transition in the $K^{\pi}=0_{2}^{+}$band, and the deduced branching fraction is $5.4 \pm 0.3 \%$. Using this value, a $B(E 2)=77 \pm 14$ W.u. is determined, consistent with but with a much smaller uncertainty compared to the previous result [16]. A similar situation occurs in the $K^{\pi}=0_{3}^{+}$ band; previously, the $2^{+} \rightarrow 0^{+}$transition was inferred but remained unobserved in this band, but the present $\beta$ decay experiment observed the transition and extracted a branching ratio of $0.79 \pm 0.09 \%$, corresponding to a $54 \pm 12$ W.u. transition. These results demonstrate that the excited $K^{\pi}=0^{+}$bands in ${ }^{124} \mathrm{Xe}$ remain very collective; at least as collective as the ground-state band. 


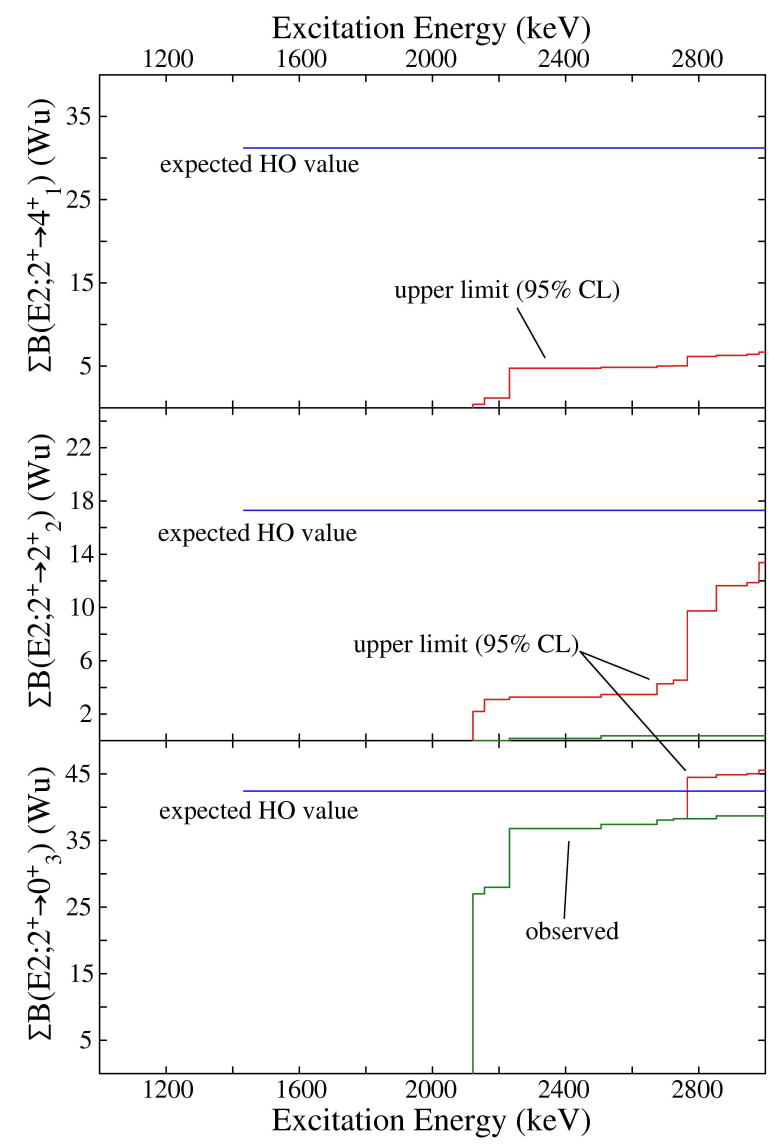

Figure 3. Cumulative sum of the observed, and upper limits of $B\left(E 2 ; 2^{+} \rightarrow 4_{1}^{+}\right)$(top), $B\left(E 2 ; 2^{+} \rightarrow 2_{2}^{+}\right)$(middle), and $B\left(E 2 ; 2^{+} \rightarrow 0_{3}^{+}\right)$(bottom) values (in W.u.) vs. excitation energy. The expected harmonic vibrator $(\mathrm{HV})$ values are indicated.

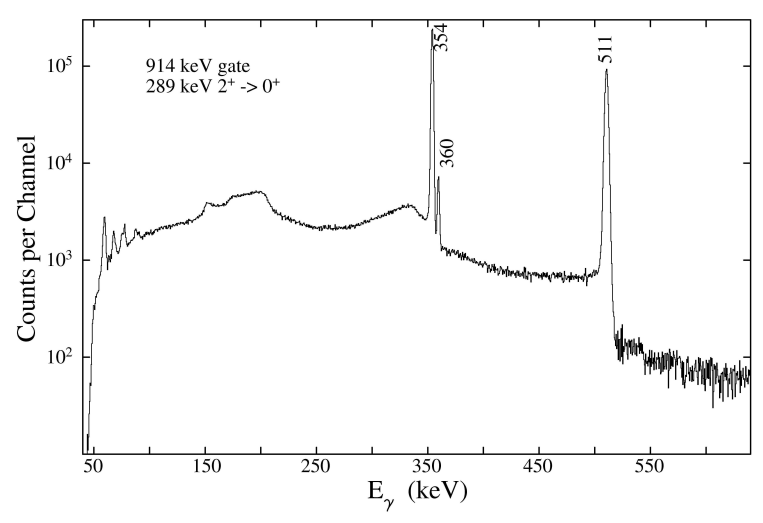

Figure 4. Portion of the $\gamma$-ray spectrum measured in coincidence with the $914-\mathrm{keV} 0_{2}^{+} \rightarrow 2_{1}^{+}$transition. The observed $360-\mathrm{keV}$ $\gamma$-ray is the $2^{+} \rightarrow 0^{+}$transition in the " $K^{\pi}=0_{2}^{+}$" band. The branching fraction for the $360-\mathrm{keV}$ transition extracted from the present data is $5.4 \pm 0.3 \%$.

\section{Complementarity and contradictions in structure assignments}

While there are many situations where data from one type of experiment can enhance the results from another, as demonstrated above, in some cases the opposite, at least at first glance, appears to occur. One of the more common examples of this can occurs when the different experiments are sensitive to different components in the wave functions. For example, Coulomb excitation is sensitive to the collective components in wave functions whereas single-nucleon transfer is sensitive to the single-particle components.

Some 15 years ago, results [21] from the ${ }^{112} \operatorname{Cd}\left(n, n^{\prime} \gamma\right)$ provided, as believed at the time, firm identification of the quadrupole-octupole-coupled (QOC) quintuplet of states. These states, with spin-parity $1^{-}-5^{-}$, formed by the coupling of the quadrupole phonon to the octupole phonon, are expected to occur in the vicinity of $E\left(2_{1}^{+}\right)+E\left(3_{1}^{-}\right)$, the sum of the excitation energies of the $2_{1}^{+}$and $3_{1}^{-}$levels. In ${ }^{112} \mathrm{Cd}$, such a situation exists as shown in Fig. 5, where the arrows are labelled with the observed $B(E 2)$ or $B(E 3)$ values in W.u. The observed enhanced $B(E 2)$ values for the $2^{-}, 3^{-}$, and $5^{-}$decays to the $3_{1}^{-}$level was taken as firm evidence for their assignment as members of the QOC quintuplet of states.

The QOC quintuplet assignment [21] in ${ }^{112} \mathrm{Cd}$ remained firm, and indeed served as a template for assignments in neighboring $\mathrm{Cd}$ isotopes [14, 22, 23]. However, very recently we have re-measured the ${ }^{111} \mathrm{Cd}(d, p){ }^{112} \mathrm{Cd}$ reaction using $22 \mathrm{MeV}$ polarized deuterons with the Q3D spectrometer at the Maier Leibnitz Laboratorium located on the joint campuses of the Technische Universität and Ludwig Maximilians Universität München. A portion of the spectrum collected at $\theta=40^{\circ}$ is shown in Fig. 6, where some of the peaks have been labelled with the corresponding excitation energies in $\mathrm{keV}$ of the levels in ${ }^{112} \mathrm{Cd}$. The most remarkable feature of the spectrum is the intensity of the $5^{-}$level at $2373 \mathrm{keV}$, and the $6^{-}$level at $2818 \mathrm{keV}$.

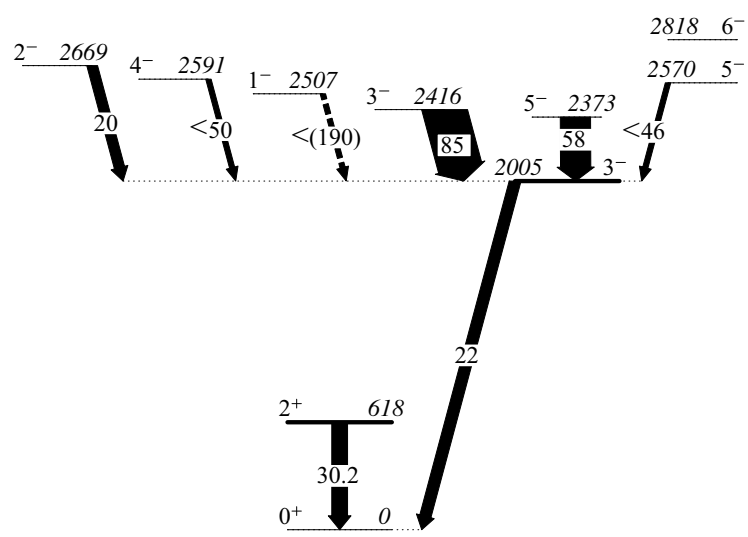

Figure 5. Partial level scheme of the low-lying negative-parity states in ${ }^{112} \mathrm{Cd}$. The $3_{1}^{-}$octupole state has an enhanced $B(E 3)$ value for decay to the ground state, establishing its one-phonon nature. The coupling of the quadrupole and octupole one-phonon states is expected to give rise to a quintuplet of states located at approximately the sum of the $2_{1}^{+}$and $3_{1}^{+}$energies. This is observed, with several of these negative-parity states possessing enhanced $B(E 2)$ values for decay to the $3_{1}^{-}$octupole phonon level, as indicated. 


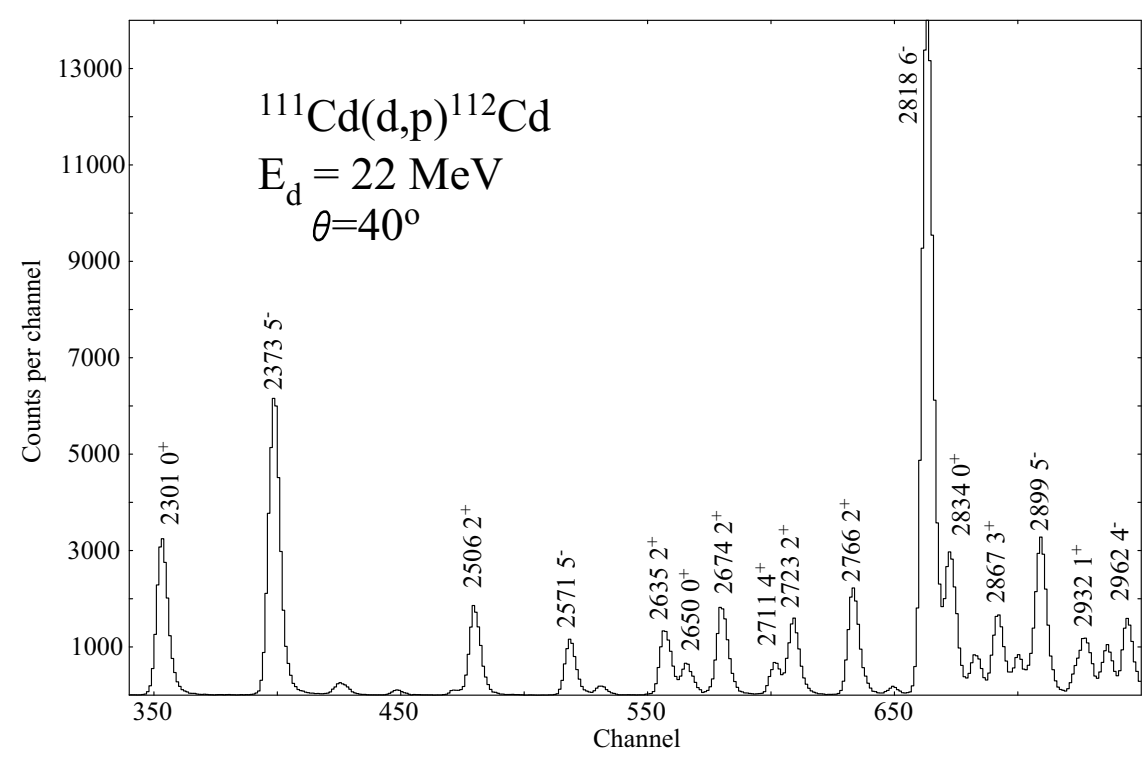

Figure 6. Portion of the spectrum observed with the ${ }^{111} \mathrm{Cd}(d, p){ }^{112} \mathrm{Cd}$ reaction using $22 \mathrm{MeV}$ deuteron beams. Because of the nature of the single-nucleon transfer reaction, only states with significant two-quasiparticle components in their wave functions of the form $j_{t} \otimes j_{t r}$, where $j_{t}$ is the target single-particle configuration and $j_{t r}$ is the configuration of the transferred particle, should be strongly populated.
These are the largest peaks in the spectrum, and from their spectroscopic strengths they are clearly dominated by the $s_{1 / 2} \otimes h_{11 / 2}$ two-quasi-neutron configuration, the $s_{1 / 2}$ configuration forming the ground state of ${ }^{111} \mathrm{Cd}$. This assignment is at odds with the identification of the $2373-\mathrm{keV} 5^{-}$ state as a member of the QOC quintuplet, but the strength observed in the $(d, p)$ reaction is irrefutable. A possible explanation lies in the suggestion that the $\mathrm{Cd}$ isotopes may represent $\gamma$-soft rotors [24]; in that picture, the $5^{-}$level would be a rotational band member based on the $3^{-}$onephonon state, and thus could be directly populated in the $(d, p)$ reaction

\section{Summary}

Complementary data obtained from a variety of reactions are vital to our understanding of the nature of nuclear excitations. Some complementary experiments, such as $\beta$ decay and Coulomb excitation (or other methods to extract nuclear level lifetimes) reinforce each other. Still other reactions may at first appear to provide contradictory results, but ultimately provide a much deeper insight to the true nature of the excitation of the nuclear system. The wide variety of reactions and probes that can be brought to bear on stable nuclei continues to provide new insights into nuclear structure, and offers the best opportunity to establish benchmarks for structure that are necessary when exploring nuclei off stability.

\section{Acknowledgements}

Much of the work presented here has formed the graduate research work of K.L. Green, A.J. Radich, and D.S. Jamieson. Their contribbution is gratefully acknowledged.

\section{References}

[1] J. Kern et al., Nucl. Phys. A 593, 21 (1995)

[2] K. Fahlander et al., Nucl. Phys. A 485, 317 (1988)

[3] F.K. McGowan et al., Nucl. Phys. 66, 97 (1965)

[4] K. Heyde et al., Phys. Rev. C 25, 3160 (1982)

[5] P.D. Duval and B.R. Barrett, Nucl. Phys. A 376, 213 (1982)

[6] T. Belgya et al., Nucl. Phys. A 607, 43 (1996)

[7] D. De Frenne and E. Jacobs, Nucl. Data Sheets 79, 639 (1996)

[8] H. Lehmann et al., Phys. Lett. B 387, 259 (1996)

[9] F. Corminboeuf et al., Phys. Rev. Lett. 84, 4060 (2000)

[10] F. Corminboeuf et al., Phys. Rev. C 63, 014305 (2000)

[11] M. Délèze et al., Nucl. Phys. A 551, 269 (1993)

[12] M. Kadi et al., Phys. Rev. C 68, 031306(R) (2003)

[13] P.E. Garrett et al., Phys. Rev. C 75, 054310 (2007)

[14] D. Bandyopadhyay et al., Phys. Rev. C 76, 054308 (2007)

[15] P.E. Garrett et al., Phys. Rev. C 78, 044307 (2008)

[16] G. Rainovski et al., Phys. Lett. B683 11 (2010)

[17] L. Coquard et al., Phys. Rev. C 83, 044318 (2011)

[18] L. Coquard et al., Phys. Rev. C 80, 061304(R) (2009)

[19] L. Coquard et al., Phys. Rev. C 82, 024317 (2010)

[20] T. Ahn et al., Phys. Lett. B 679, 19 (2009)

[21] P.E. Garrett et al., Phys. Rev. C 59, 2455 (1999)

[22] A. Gade et al., Phys. Rev. C 66, 034311 (2002)

[23] J.C. Batchelder et al., Phys. Rev. C 80, 054318 (2009)

[24] P.E. Garrett and J.L. Wood, J. Phys. G 37, 064028 (2010); corrigendum ibid., 069701 
